\title{
Recognition of Japanese Phonographic Kana (Hiragana) and Logographic Kanji Characters by Passive Finger Tracing
}

\author{
Hikari Yamashita \\ Faculty of Education, Ehime University, Matsuyama, Japan \\ Email: yamabcd@ed.ehime-u.ac.jp \\ Received 16 January 2014; revised 13 February 2014; accepted 12 March 2014 \\ Copyright (C) 2014 by author and Scientific Research Publishing Inc. \\ This work is licensed under the Creative Commons Attribution International License (CC BY). \\ http://creativecommons.org/licenses/by/4.0/

c) (i) Open Access

\begin{abstract}
The present study assessed the ability of normal Japanese adults to recognize kanji and hiragana characters through passive finger tracing without visual cues. We tested fifty-six right-handed Japanese university students using the left or right hand. Participants recognized approximately $50 \%$ of 44 kanji characters, regardless of the hand they used. The results are consistent with previous findings in Chinese speakers. In contrast, the average correct response to the 44 hiragana was almost $80 \%$, again irrespective of hand. The results are discussed in terms of differential processing systems for Japanese writing and of differential cerebral hemispheric specializations.
\end{abstract}

\section{Keywords}

Japanese Reading; Laterality; Kana; Kanji; Finger Tracing

\section{Introduction}

Finger tracing or "kusho behavior" is writing-like-behavior performed in the air, on a desk, or on one's lap without pen and paper when writing Chinese characters (Kanji) that is often seen in Chinese and Japanese people. It is considered to provide evidence that orthographic information processing involves components of "graphomotoric coding" or "kinesthetic memory of writing" (Hoosain, 1991; Matsuo, Kato, Okada, Moriya, Glover, \& Nakai, 2003; Sasaki \& Watanabe, 1983, 1984). A phenomenon similar to tracing is also observed in patients with language disorders caused by brain damage. Patients suffering from alexia without agrahia (or pure alexia) following damage to the left occipital cortex and the splenium of the corpus callosum can often "read" the letters by tracing voluntarily or passively (others take the hand and move) with their fingers. This "kinesthetic facilitation effect” suggests that alexic patients can identify the letters through kinesthetic feedback (e.g., 
Friedman, Ween, \& Albert, 1993; Fukunaga, Hattori, Tagawa, \& Ubukata, 2010; Kashiwagi \& Kashiwagi, 1989; Seki, Yajima, \& Sugishita, 1995). One possible interpretation of this phenomenon is that kinesthetic information from the finger tracing of the right hand is transferred through the intact part of the corpus callosum to the language-related areas of the left hemisphere (Seki et al., 1995).

Yim-Ng, Varley, and Andrade (2000) reported an interesting study of the links from the motor representation of writing to the reading system in healthy Chinese speakers. They asked right-handed, blindfolded Chinese volunteers to identify Chinese characters (kanji) through passive tracing movements guided by the experimenter, using the fingers of the right hand. The participants were able to recognize on average approximately $70 \%$ of the characters. In other words, normal participants could recognize Chinese characters solely by kinaesthetic feedback without visual information. These research findings also support the existence of graphomotoric coding (information related to writing movement generated by the visuospatial representations of characters) in native users of Chinese. We were interested in investigating whether this phenomenon also occurred with native speakers of Japanese when using the Chinese characters (kanji) in the same way. However, in Japanese, there is an important problem that arises from the characteristics of the notation. The Japanese writing system has two different types of character, kanji and kana. Kanji characters are logographic in nature and derived from imported Chinese characters. Nowadays only around 2000 kanji characters are in common use. In contrast, phonographic kana characters were developed originally in Japan. Kana has two types of character, hiragana and katakana, both of which have 46 basic characters. Because they were devised by cursive forms (hiragana) and abbreviations (katakana) of kanji, the configurations of kana characters are simpler than the majority of kanji characters. Kana is syllabic system, in which a single pronunciation is always represented by a pair of hiragana and katakana characters. In contrast, most of the kanji characters used in everyday Japanese have two or more different pronunciations (Habein, 1984; Henshall and Kawai, 2004; Sato, 2013). In fact, most Japanese sentences contain both kanji (e.g., most nouns and the stems of most verbs and adjectives) and hiragana (e.g., most function words, the inflections of verbs and adjectives, and some content words), while some additionally use katakana (e.g., loanwords from Western languages, foreign personal names). Figure 1 shows an example of the Japanese sentence notation.

Since kana has a highly regular orthography, most Japanese children quickly master kana reading and writing in the first grade of elementary school (at the age of $6-7$ years). They begin to learn kanji after having learnt kana. By the end of the sixth grade, Japanese children should have mastered about 1000 kanji characters. However, the relative readability of kana and kanji is affected by various factors including familiarity and frequency with which they are written at the level of words and sentences (Besner \& Hildebrandt, 1987; Chikamatsu, Yokoyama, Nozaki, Long, \& Fukuda, 2000; Sugishita, Otomo, Kabe, \& Yunoki, 1992; Ukita \& Ukita, 1996). Japanese aphasia and alexia patients are known to show various types of dissociation between the ability to process kana and kanji. Among these patterns, there are more case reports of alexia specific to, or predominant for, kana rather than kanji characters (Sasanuma, 1975; Yamadori, 1998). This interesting phenomenon has been reported in many studies, and two approaches have been taken to explain it. One approach proposes that both kanji and kana are processed by a common set of essentially left hemisphere regions, but rely on different intra-hemispheric neuronal circuits. For example, Iwata (1984) proposed that the semantic processing of reading kanji depends on the left posterior inferior temporal area, while the phonological reading of kana is mediated by the left angular gyrus. The other approach proposes that kana characters are processed mainly by the left hemisphere, but kanji characters may be processed by both left and right hemispheres owing to the greater ability of the right hemisphere to process visual features. In particular, studies using unilateral tachistoscopic presentation have demonstrated that although the right cerebral hemisphere is more efficient than the left hemisphere at processing single kanji characters (Hatta, 1978; Nakagawa, 1994), there is a reverse tendency when processing two-cha-

\section{私は 毎日 ビールを 飲みます。 \\ Watashi wa mainichi biru o nomimasu.}

\section{'I drink beer every day.'}

Figure 1. An example of Japanese sentence notation. Double underlined are kanji, underlined are katakana, and others are hiragana. 
racter kanji words or kanji-kana mixed words (Hatta, 1978). Results that were totally similar to these Japanese studies were found in Chinese experiments using Chinese characters and words (Cheng \& Yang, 1989; Tzeng, Hung, Cotton, \& Wang, 1979). However, there is also a radical opinion that denies the essential difference between kanji and kana reading, based on a more careful examination of various aphasic patients (Sugishita et al., 1992).

The aims of the present study were three-fold. The first was to evaluate the ability of normal Japanese adults to recognize Japanese characters through passive tracing without the aid of visual input. The second was to examine whether there was a difference in the readability of kana and the kanji through passive tracing. A particularly notable finding in the Japanese pure alexia patients was that the kinesthetic facilitation effect by finger tracing was greater in kana than kanji (Torii, Fukuta, \& Koyama, 1972; Iwata, 1977). The third aim was to examine whether reading performance differed between the right (dominant) and left (nondominant) hand. If the right hemisphere participates in the reading of kanji, then a difference may occur for reading with the left and right hands. Furthermore, another interesting question is whether the difference between the left and right hands occurred in kana recognition.

\section{Method}

\subsection{Design}

To exclude an effect of order of hand use, we adopted a $2 \times 2$ repeated measures design, with the hand (right and left) used as a between-subjects factor and stimuli (kana and kanji) as a within-subjects factor.

\subsection{Participant}

The participants were 56 right-handed Japanese university students (28 males and 28 females: mean age $20.5 \pm$ 1.5 years; age range 18 - 24 years). Right handedness was determined using the H. N. Handedness Inventory (HNI: Hatta \& Nakatsuka, 1974). The HNI, which has been widely used in Japanese handedness-related studies, consists of 10 items regarding hand preference for daily activities. It assigns a score of +1 when the right hand is used, -1 when the left hand is used, and 0 when the two hands are used in approximately equally. Therefore the HNI handedness score ranges from -10 to +10 . The criterion for right handedness is a score $>+8$.

Each participant was randomly assigned by sex to one of two hand groups, each with 14 men and 14 women, on the basis of the hand to be used in the tracing task: left hand (mean age $=20.4$ years, $S D=1.6$ ) and right hand (mean age $=20.7$ years, $S D=1.4$ ).

All the procedures in this study conformed to the code of ethics and conduct of the Japanese Psychological Association. All participants gave informed written consent for participation.

\subsection{Materials}

As mentioned before, there are two types of Japanese phonographic kana, termed hiragana and katakana, of which the former is the more commonly used. Only hiragana characters were used in the present study. Fortyfour of the 46 hiragana characters were used (see Table 1; two special characters were excluded). The number of kanji characters in common use is nearly 2000, far greater than the number of kana characters. Further, there is considerable difference in the frequency of use and familiarity of the kanji characters (Besner \& Hildebrandt, 1987; Chikamatsu et al., 2000; Sugishita et al., 1992). In an effort to equalize the difficulty of reading kanji and kana, we selected 44 kanji characters that are taught during the first grade in elementary schools, with reference to the method of Sugishita et al. (1992). These characters are very commonly used in Japanese everyday life (Table 2).

\subsection{Procedures}

Each participant sat blindfolded at a table in a normal writing posture and was asked to put their index finger of the right or left hand on the table. The experimenter stood behind the participant and held their hand, guiding the finger to trace a character printed on a piece of paper. The size of all characters was identical $(4 \mathrm{~cm} \times 4 \mathrm{~cm})$. The experimenter instructed each participant as follows: "I would like you trace a letter with your right (or left) hand. The letter to trace is a basic kanji character or a hiragana letter. Please tell me what the letter which you traced 
Table 1. Phonographic hiragana stimuli (number of strokes).

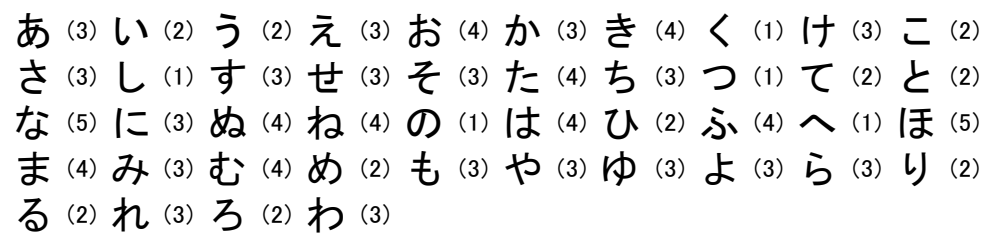

Table 2. Logographic kanji stimuli (number of strokes).

入 (2) 女 (3) 川 (3) 子 (3) 小 (3) 大 (3) 山 (3) 円 (4) 王 (4) 手 (4)
日 (4) 水 (4) 生 (5) 田 (5) 立 (5) 出 (5) 本 (5) 目 (5) 糸 (6) 気 (6)
休 (6) 字 (6) 先 (6) 竹 (6) 名 (6) 年 (6) 早 (6) 耳 耳 $^{\text {(6) 虫 (6) 赤 (7) }}$
足 (7) 男 (7) 車 (7) 町 (7) 見 (7) 青 (8) 雨 (8) 学 (8) 金 (8) 空 (8)
林 (8) 音 (9) 草 (9) 校 (10)

was. The same letter may appear more than once.” The typical sequence of strokes used to write each character was used, and the experimenter controlled the tracing speed to be consistent with the natural writing speed. After each complete trace, the experimenter asked the participants what character they had traced, and their responses were recorded immediately. There was no time limit between the end of tracing and the response. The kanji and kana stimuli were tested in separate sessions. The order of sessions and the presentation order of stimuli in each session were randomized. Each character was presented only once. A total of 88 trials were conducted. After finger tracing sessions, each stimulus character was presented visually to the participants, and they were instructed to read them aloud in order to check for difficult or unknown characters.

\subsection{Measures}

The mean percentage of correct responses for each participant in each condition was taken as an index of the accuracy of identification (reading). For a kanji character with two or more pronunciations, any of these pronunciations was judged to be a correct answer. The erroneous responses were categorized into two types: 1) no response (including “I could not read it”), 2) misidentifications with other letters (including partial identification or partial substitution).

\subsection{Data Analyses}

The mean percentage of correct responses for the left- and right-hand groups on the kanji and hiragana tasks were analyzed using a two-way mixed model ANOVA, with hand used (group) as a between-subjects factor, and tasks (kanji, kana) as a repeated-measures, within-subjects factor.

One factor that could potentially account for a discrepancy between kanji and kana is the difference in visuospatial complexities of the two types of characters. Sugishita et al. (1992) suggested that the number of strokes required to write each character provides a useful measure of complexity. According to this proposal, no kana character has more than five strokes. In contrast, the kanji characters employed here had between 2 and 10 strokes. Therefore, some of the kanji characters could be considered to be visuospatially more complex than the kana characters. In order to assess the possible effects of visuospatial complexity on reading performance, an additional analysis was conducted using the method previously employed by Sugishita et al. (1992) when studying the reading abilities of Japanese aphasic patients. The kanji characters were classified into two subsets: a high complexity set (HC-kanji) comprising those formed with many strokes (6 to 10), and a low complexity set (LC-kanji) comprising those written with few strokes (2 to 5). In the present study, the high complexity set included 26 characters, and the low complexity set included 18 characters. A one-way within-subjects ANOVA was carried out on the mean percentage of correct responses between the three groups of characters.

\section{Results}

Table 3 shows the mean percentage of correct responses for left-hand and right-hand groups on the kanji and 
kana tasks.

A two-way mixed model ANOVA (Group $\times$ Task) indicated a significant main effect of task $[F(1,53)=$ 192.63, $p<.001$, partial $\left.\eta^{2}=.784\right]$. In contrast, the main effect of group $[F(1,53)=.52]$ and the Group $\times$ Task interaction $[(F(1,53)=1.35, p=.25]$ were not significant. This result indicates that hiragana characters are recognized more accurately than kanji characters regardless of the hand used. From an analysis of errors, the proportion of no response was greater than $70 \%$ in the kanji task (73.7\% in the left-hand group; $70.0 \%$ in the righthand group). There were few misidentifications with other letters (including partial identification or partial substitution: $26.3 \%$ in the left-hand groups; $30.0 \%$ in the right-hand group).

On the other hand, there were around $50 \%$ of no responses in the hiragana task (51.3\% in the left-hand group; $54.6 \%$ in the right-hand group). There were many misidentifications in which the character was confused with other letters (48.7\% in the left-hand group; $45.4 \%$ in the right-hand group).

Table 4 shows the mean percentage of correct responses to hiragana and the two types of kanji stimuli for merged left- and right-hand groups. A one-way ANOVA showed a significant difference $[F(2,110)=146.02, p$ $<.001$, partial $\left.\eta^{2}=.799\right]$. Tukey HSD tests $(p<.05)$ indicated significant differences between all three stimuli types. The hiragana stimulus were most easily recognized, followed by the low and high complexity kanji. Finally, on the visual reading task after the experiment, all participants were immediately able to read aloud all kanji and hiragana stimuli.

\section{Discussion}

The present study assessed the ability of normal Japanese adults to recognize kanji and kana (hiragana) characters through passive tracing without visual information. Participants were able to identify an average of $50 \%$ of kanji stimuli, regardless of the hand used. In contrast, the average correct responses to the hiragana characters reached almost $80 \%$, again regardless of hand used. Because the greater visuospatial complexity of the kanji characters may have affected performance, the data were reanalyzed with the kanji stimuli classified into high and low complexity subsets on the basis of the number of strokes needed to form each character. Comparison of performances for the high and low complexity kanji stimuli revealed significantly greater accuracy for the low complexity kanji stimuli. This difference is consistent with results reported by Yim-Ng et al. (2000). However, performance was still better for the hiragana stimuli than for the low complexity kanji stimuli. The greater rea-

Table 3. Means, standard deviations, and ranges for percentage of correct recognition of kana and kanji characters by finger tracing for each hand group.

\begin{tabular}{ccccc}
\hline & \multicolumn{4}{c}{ Groups } \\
\cline { 2 - 5 } & \multicolumn{2}{c}{ LH $(\mathrm{n}=28)$} & \multicolumn{2}{c}{ RH $(\mathrm{n}=28)$} \\
\cline { 2 - 5 } & kanji & hiragana & kanji & hiragana \\
\hline Mean & 50.2 & 78.3 & 53.6 & 81.4 \\
SD & 10.0 & 8.6 & 14.3 & 8.1 \\
Range & $22.7-68.2$ & $59.10-90.9$ & $22.7-79.6$ & $61.4-95.5$ \\
\hline
\end{tabular}

Table 4. Means and standard deviations for percentage of correct recognition for hiragana and high- and low-complexity kanji stimuli.

\begin{tabular}{cccc}
\hline & \multicolumn{3}{c}{ Stimulus Type } \\
\cline { 2 - 4 } & HC kanji & LC kanji & hiragana \\
\hline Mean & 45.7 & 60.1 & 79.8 \\
SD & 15.1 & 12.7 & 8.5 \\
\hline
\end{tabular}

Note. Abbreviations: HC kanji = high-complexity kanji stimuli; LC kanji = low-complexity kanji stimuli; hiragana = hiragana stimuli. 
dability of kana in comparison with kanji presented visually is often explained by a frequency effect (Besner \& Hildebrandt, 1987; Sugishita et al., 1992). However, the kanji stimuli that we used included only characters that are frequently used in everyday Japanese life. The fact that every participant was able easily to read aloud all visually shown kanji stimuli provides evidence of the high frequency of our kanji character set. These results cannot be interpreted in terms of either the configurational complexity or the low frequency of kanji characters.

One possible explanation of differences between the performances with the present kanji and hiragana stimuli is the difference in the degree of reliance on graphomotoric coding. Based on the findings of brain imaging studies of reading and writing processes and case studies of Japanese pure alexia patients, Nakamura and Kouider (2003) put forward the hypothesis that motor representations (or kinesthetic memory) of kanji characters are more closely associated with their visual images and are not as effectively interconnected with phonology. On the other hand, motor representations of kana characters are likely to link more directly with the phoneme system. This is a hypothesis that merits examination in the future studies of reading through the kinesthetic channel. However, we cannot completely exclude the possibility that the fact there are only 46 hiragana characters (of which only 44 were actually used) provides clues to their identification.

Another important finding was that for either hiragana or kanji stimuli, recognition performance using the left hand did not differ significantly from that using the right hand. Logically, coding through the right hand, for which information directly accesses the left hemisphere, should result in superior reading by the left hand. Furthermore, there may be a kinesthetic memory of skilled writing in the left brain because the right hand is better at writing. On the other hand, if kanji characters were processed by the right hemisphere as well as the left, then recognition performances of the left and right hands might be expected to differ in kanji and hiragana tasks. However, we were not able to find any evidence of a right-hemisphere advantage in the processing of the single kanji characters in the present experiment. However, this fact is rather beneficial in relation to the use of tracing for the rehabilitation of reading disorders. If word identification performance using the nondominant hand was substantially lower than that of the dominant one, then rehabilitation using finger tracing might be ineffective for patients with sensory or movement disorders on the dominant side. The present results suggest the possibility that tracing with the nondominant hand may be as effective as the dominant hand for patients with reading disorders.

One limitation of this study is that the number of kanji stimuli was limited to 44 characters that are taught in the first grade of elementary school, out of the nearly 2000 characters in common use. In addition to high frequency and familiarity, those kanji characters are morphologically simpler than less frequently used kanji characters. Therefore, it may to some extent be unwarranted to claim that the present results reflect a general tendency in kana and kanji recognition. These limitations notwithstanding, the present study has added new knowledge about the importance of graphomotoric coding in the Japanese writing systems.

\section{Acknowledgements}

This research was supported by a Grant-in-Aid for Scientific Research (C) from JPSP to HY (No. 24530873).

\section{References}

Besner, D., \& Hildebrandt, N. (1987). Orthographic and Phonological Codes in the Oral Reading of Japanese Kana. Journal of Experimental Psychology: Learning, Memory, and Cognition, 13, 335-343. http://dx.doi.org/10.1037/0278-7393.13.2.335

Cheng, C. M., \& Yang, M. J. (1989). Lateralization in the Visual Perception of Chinese Characters and Words. Brain and Language, 36, 669-689. http://dx.doi.org/10.1016/0093-934x(89)9009393-X

Chikamatsu, N., Yokoyama, S., Nozaki, H., Long, E., \& Fukuda, S. (2000). Japanese Logographic Character Frequency List for Cognitive Science Research. Behavior Research Methods, Instruments, \& Computers, 32, 482-500. http://dx.doi.org/10.3758/bf03200819

Friedman, R., Ween, J. E., \& Albert, M. L. (1993). Alexia. In K. M. Heilman, \& E. V. Valenstein (Eds.), Clinical Neuropsychology (pp. 37-62). New York: Oxford University Press.

Fukunaga, S., Hattori, F., Tagawa, K., \& Ubukata, S. (2010). Investigation of Kanji and Kana Reading Discrepancies in a Pure Alexia Case: Comparison of Oral Reading and Kinesthetic Facilitation Reading of Single Kanji and Kana. Higher Brain Function Research, 30, 96-101. http://dx.doi.org/10.2496/hbfr.30.96

Hatta, T. (1978). Recognition of Japanese Kanji and Hiragana in the Left and Right Visual Fields. Japanese Psychological Research, 20, 51-59. http://dx.doi.org/10.1016/0028-3932(77)90073-2 
Hatta, T., \& Nakatsuka, Z. (1974). Hatta-Nakatsuka Handedness Test. In Ohno, S. (Ed.), Festschrift to Prof. Ohnishi. K. (pp. 224-247). Osaka: Osaka City University Press.

Habein, Y. S. (1984). The History of the Japanese Written Language. Tokyo: University of Tokyo Press.

Henshall, K., \& Kawai, J. (2004). Welcome to Japanese: A Beginner's Survey of the Language. Boston: Tuttle Publishing.

Hoosain, R., (1991). Psycholinguistic Implications for Linguistic Relativity: A Case Study of Chinese. Hove: Lawrence Erlbaum.

Iwata, M. (1977). Neuropsychological Aspects of Pure Alexia Syndrome. Shinkei Shinpo, 21, 930-940.

Iwata, M. (1984). Kanji versus Kana: Neuropsychological Correlates of the Japanese Writing System. Trends in Neuroscience, 8, 290-293. http://dx.doi.org/10.1016/s0166-2236(84)80198-8

Kashiwagi, T., \& Kashiwagi, A. (1989). Recovery Process of a Japanese Alexic without Agraphia. Aphasiology, $3,75-91$. http://dx.doi.org/10.1080/02687038908248977

Matsuo, K., Kato, C., Okada, T., Moriya, T., Glover, G. H., \& Nakai, T. (2003). Finger Movements Lighten Neural Loads in the Recognition of Ideographic Characters. Cognitive Brain Research, 17, $263-272$. http://dx.doi.org/10.1016/s0926-6410(03)00114-9

Nakagawa, A. (1994). Visual and Semantic Processing in Reading Kanji. Journal of Experimental Psychology: Human Perception and Performance, 20, 864-875. http://dx.doi.org/10.1037/0096-1523.20.4.864

Nakamura, K., \& Kouider, S. (2003). Functional Neuroanatomy of Japanese Writing Systems. Aphasiology, 17, 667-683. http://dx.doi.org/10.1080/02687030344000076

Sasaki, M., \& Watanabe, A. (1983). An Experimental Study of Spontaneous Writing-Like Behavior (“Kusho”) in Japanese. The Japanese Journal of Educational Psychology, 31, 273-282.

Sasaki, M., \& Watanabe, A. (1984). Cultural Origin of “Kusho” Behavior. The Japanese Journal of Educational Psychology, 32, 182-190.

Sasanuma, S. (1975). Kana and Kanji Processing in Japanese Aphasics. Brain and Language, 2, 369-383. http://dx.doi.org/10.1016/s0093-934x(75)80077-0

Sato, E. (2013). Mienaimoji to Mierumoji. Tokyo: Sanseido.

Seki, K., Yajima, M., \& Sugishita, M. (1995) The Efficacy of Kinesthetic Reading Treatment for Pure Alexia. Neuropsychologia, 33, 595-609. http://dx.doi.org/10.1093/neucas/1.2.139-y

Sugishita, M., Otomo, K., Kabe, S., \& Yunoki, K. (1992) A Critical Appraisal of Neuropsychological Correlates of Japanese Ideogram (KANJI) and Phonogram (KANA) Reading. Brain, 115, 1563-1585. http://dx.doi.org/10.1093/neucas/6.4.292-a

Torii, H., Fukuta, T., \& Koyama, Y. (1972) A Report of Three Cases of Alexia without Agraphia Due to Cerebrovascular Lesion and Semiological Consideration. Psychiatria et Neurologia Japonica, 74, 546-576.

Tzeng, O., Hung, D., Cotton, B., \& Wang, W. (1979) Visual Lateralization Effect in Reading Chinese Characters. Nature, 282, 499-501. http://dx.doi.org/10.1038/282499a0

Ukita, J., \& Ukita, H. (1996). Effects of Types of Written Forms on Word Reading Time of Aphasic Patients. Higher Brain Function Research, 16, 331-335. http://dx.doi.org/10.2496/apr.16.331

Yamadori, A. (1998) Aphasia in Ideograph Readers: The Case of Japanese. In P. Coppens, Y. Lebrun, \& A. Basso (Eds.), Aphasia in Atypical Populations (pp. 143-174). Mahwah, NJ: Laurence Erlbaum Associates.

Yim-Ng, Y. Y., Varley, R., \& Andrade, J. (2000) Contribution of Finger Tracing to the Recognition of Chinese Characters. International Journal of Language and Communications, 35, 561-571. http://dx.doi.org/10.1080/136828200750001296 